\title{
Underachievement Ein Blick in die Black Box eines irritierenden Phänomens
}

\section{Margrit Stamm}

Dieser Aufsatz präsentiert die Ergebnisse einer Studie zu überdurchschnittlich begabten Underachievern. Basierend auf den Daten zweier Schweizer Längsschnittstudien wurden zwei überdurchschnittlich begabte Subgruppen, die Underachiever (definiert als Jugendliche mit IQ-Prozentrang $\geq 90$ und Schulleistungsprozentrang in Deutsch/Mathematik 550) und die Achiever (IQ-Prozentrang $\geq 90$ und Schulleistungsprozentrang in Deutsch/Mathematik $\geq 75$ ) herausgefiltert und in Bezug aufsozial-strukturelle Merkmale, Schuleinstellungen und Karrierepläne untersucht. Die Hauptbefunde verweisen einerseits auf klare Differenzen zwischen Achievern und Underachievern. Diese sind in erster Linie männlichen Geschlechts, stammen aus eher bildungsnahen Familien, sind weniger leistungsmotiviert und haben grössere Probleme mit der Selbstregulation. Andererseits schwänzen sie häufiger die Schule und berichten über deutlich schlechtere Lehrerbeziehungen als die Achiever. Damit verweisen diese Befunde auf die Notwendigkeit, Underachievement nicht nur mit Blick auf die individuelle, sondern auch auf die institutionelle Perspektive in den Blick zu nehmen.

Underachiever haben Lehrkräfte und Eltern schon immer irritiert. Auch die Medien portraitieren gerne smarte Schülerinnen und Schüler, die sich trotz hohem intellektuellem Potenzial nur mit knapp genügenden Noten über die Runden schlängeln oder gar eine Klasse wiederholen müssen. Im Unterschied zur medialen Diskussion präsentiert sich der wissenschaftliche Diskurs zurückhaltend und kontrovers. So liegen kaum eindeutige Befunde zur Prävalenz, Entstehung und Charakteristik von Underachievement vor, und seine Existenz wird sogar gelegentlich bezweifelt oder als statistisches Artefakt bezeichnet (Anastasi, 1976). Der Grund liegt vor allem darin, dass Leistungs- und Begabungsindikatoren nur mit einem gemeinsamen Varianzanteil von 25\% bis 50\% korrelieren. Deshalb muss angenommen werden, dass noch andere Faktoren wie etwa Persönlichkeits- oder Kontextmerkmale eine Rolle spielen, was die Bedeutung der Intelligenz als Prädiktorvariable deutlich schmälert. Gegenargumente liegen ebenfalls vor. So führen Hanses und Rost (1998) oder Holling und Preckel 
(2005) aus, dass der Grund für die lediglich moderaten Korrelationen darin liege, dass bestimmte Schülergruppen eben erwartungswidrige Leistungen (niedrigere oder höhere) zeigten und dass sich Underachiever in verschiedenen Untersuchungen von anderen Schülergruppen in persönlichkeitsbezogenen, familiären oder schulischen Variablen unterscheiden würden.

Tatsache ist jedoch, dass es Underachiever gibt und deren empirische Reliabilität in den letzten vierzig Jahren vielfach unter Beweis gestellt (Hanses \& Rost, 1998; Raph, Goldberg \& Passow, 1966; McCall, Evahn \& Kratzer, 1992; Stamm, 2005a) und auch in der Biografieforschung mit berühmten Beispielen konturiert worden ist, man denke beispielsweise an Winston Churchill, Alvar Edison oder Bill Gates (Prause, 1996). In der Vergangenheit sind zwar eine Vielzahl schulbezogener wie auch kausaler und korrelativer Faktoren diskutiert worden, viele Fragen jedoch bleiben offen: Warum gibt es überdurchschnittlich begabte Schülerinnen und Schüler, die so wenig leisten, obwohl sie doch das intellektuelle Potenzial dazu hätten? Welche Charakteristika unterscheiden sie von überdurchschnittlich Begabten, die gute bis sehr gute Schulleistungen vorweisen können?

Solche Fragen sind Thema dieses Aufsatzes: Auf der Datenbasis zweier Schweizer Längsschnittprojekte stellt er die Befunde einer Teilstudie vor, welche überdurchschnittlich begabte Underachiever überdurchschnittlich begabten Achievern gegenüber stellt und nach charakteristischen Gemeinsamkeiten und Unterschieden fragt.

\section{Zum Forschungsstand von Underachievement}

Im deutschsprachigen Europa ist Underachievement nur rudimentär erforscht und vorwiegend auf Persönlichkeitsprofile (Rost \& Hanses, 1997) oder Lehreridentifikation (Hanses \& Rost, 1998) sowie auf einige ältere theoretische Arbeiten beschränkt (Flammer \& Keller, 1978; Wahl, 1975; Weinert \& Petermann, 1980). Im angloamerikanischen Sprachraum wird die Thematik breiter, aber ebenso kontrovers, diskutiert. Strittig ist zunächst einmal die Definition von Underachievement, weil je nach Definitionsgrundlage unterschiedliche Anteile von Underachievern in der Gruppe der Hochbegabten resultieren. Während operationale Definitionen Underachievement in der Regel als Diskrepanz von Performanz und Kompetenz (z. B. Baum, Renzulli \& Hébert, 1995; Dowdall \& Colangelo, 1982; Whitmore, 1980) oder als Diskrepanz von Performanz, Kompetenz und Intelligenz (Colangelo, Kerr, Christensen \& Maxey, 1993; Durr, 1964; Shaw \& McCuen, 1960; Supplee, 1990) definieren und Underachievement auf Schülerinnen und Schüler zwischen dem IQ-Prozentrang von $\geq 88$ und $\geq 95$ und einem Schulleistungsprozentrang von $\leq 50$ in bestimmten Fächern einschränken, geben konzeptuelle Definitionen keine exakten Kriterien vor. Sie setzen lediglich fest, dass Schülerinnen und Schüler dann als Underachiever zu be- 
zeichnen sind, wenn die Schulleistungen nicht ihren Fähigkeiten entsprechen. Es versteht sich somit von selbst, dass sich solche konzeptuellen Definitionen ausschliesslich am Potenzial orientieren und deshalb für alle, nicht nur für die überdurchschnittlichen Lernniveaus, angewendet werden können. Dies ist der Grund, warum gewisse Studien, von einem relativ grossen, bis zu 50\% umfassenden Anteil an Minderleistern ausgehen (Peters, Grager-Loidl \& Supplee, 2000; Richert, 1991).

Bestimmte operationale Ansätze ziehen zur Voraussage von Leistungen über Fähigkeiten regressionsanalytische Modelle heran (McCall, Evahn \& Kratzer, 1992; Smith, 2003). Dabei hat sich eingebürgert, Kinder und Jugendliche als Underachiever zu klassifizieren, wenn ihre Residuen (d.h. die Abweichungen zwischen tatsächlichen und erwarteten Werten) bei der Leistungsvorhersage einen bestimmten Grenzwert um zwei Residualabweichungen übersteigen. Obwohl diesem Verfahren wesentliche Vorteile attestiert werden, die gemäss Klauer (1990) oder Smith (2003) vor allem darin liegen, dass ein erwarteter Wert für jeden Schüler individuell bestimmbar wird, wurde es verschiedentlich kritisiert, dass es lediglich den $\alpha$-Fehler berücksichtige, den $\beta$-Fehler jedoch ausser acht lasse. Während der $\alpha$-Fehler die fälschliche Klassifikation von erwartungsgemäss minderleistenden Schülern als erwartungswidrig beinhaltet, klassifiziert der $\beta$ Fehler tatsächlich erwartungswidrig minderleistende Schüler als erwartungsgemäss (vgl. dazu auch Sparfeldt \& Schilling, 2006). Basierend auf dieser Kritik entwickelte Klauer (1990) ein Modell, das sowohl den $\alpha$-als auch den $\beta$-Fehler kontrolliert. Vergleicht man regressionsanalytische und IQ-basierte Verfahren miteinander, so zeigen sich grundlegende Unterschiede: Während regressionsanalytische Methoden das gesamte Fähigkeitsspektrum in den Blick nehmen und nicht auf ausschliesslich hochbegabte Individuen beschränkt sind, fokussieren IQ-basierte Cut-off-Werte ausschliesslich auf den hoch begabten Underachiever (Holling \& Preckel, 2005).

Aber nicht nur an der Definitionsfrage zeigt sich, dass dem Underachievement-Konstrukt keine systematische und allgemein akzeptierte Betrachtungsweise eigen ist. Ebenso zufällig geblieben sind Antworten auf Fragen, welche die Invarianz von Minderleistung gegenüber Populationsunterschieden betreffen (Ist Underachievement unabhängig vom Geschlecht, von der sozialen Herkunft oder vom Schultyp?), die Stabilität gegenüber der Substitution von Ausgangsvariablen (Ist Underachievement unabhängig von der Auswahl der Prädiktoren und Kriterien oder gibt es so viele Arten von Underachievement wie es Definitionen gibt?) oder die zeitliche Konstanz (Ist Underachievement über die Schulzeit hinweg stabil oder trifft dies nur für bestimmte Etappen zu?).

Ein ungelöstes Problem ist auch die Frage nach den Faktoren, die mit Underachievement verbunden sind. Trotzdem gibt es einen gemeinsamen Tenor der internationalen Forschungsarbeiten. Dieser fokussiert auf Underachievement als multikausalem Phänomen, das vor allem durch die Faktorenbündel Persönlichkeit, Familie und Schule indikatorisiert wird (Baker, Bridger \& Evans, 1998). 
Am besten untersucht sind Persönlichkeitsvariablen der Motivation und des Selbstkonzepts. Ein negatives Selbstkonzept ist eines der am häufigsten beschriebenen Charakteristika (Diaz, 1998; Ford, 1996; Hanses \& Rost, 1998; McCoach \& Siegle, 2003). Einen besonderen Stellenwert räumt die Forschung den elterlichen Faktoren ein, die mit dem Underachievement-Verhalten des Kindes zu interagieren scheinen, ohne dass allerdings bislang Klarheit über die Art und Weise oder die Gründe der Einflussnahme gewonnen werden konnte. So liegen Arbeiten vor, die Belege für Überbehütung (Weiner, 1992) oder für besonders hohe Erwartungen in und übergrosse Sorge um schulische Leistungen liefern (Brown et al., 1993). Andererseits gibt es auch klare Hinweise auf autoritäre (Freeman, 1992; Taylor, 1994) oder von extremer Permissivität, distanzierter Interaktion und geringer Emotionalität gezeichnete Erziehungsstile (Jeon \& Feldhusen, 1993). Übereinstimmend sind die Befunde, wonach die Konfliktintensität in Familien von Underachievern höher ist als in Familien von Achievern (vgl. zusammenfassend Reis et al., 1995). Unter dem Stichwort des Hidden Underachievements (Baker, Bridger \& Evans, 1998) gilt seit einigen Jahren auch hochbegabten Underachievern aus sozio-ökonomisch benachteiligten Milieus und aus anderen Kulturen erhöhte Aufmerksamkeit. Angenommen wird dabei, dass gerade begabte Schülerinnen und Schüler mit Minoritätshintergrund besonders häufig unauffällige Minderleister sind. Der Grund liegt darin, dass ihnen durch ihre Herkunft viele Nachteile erwachsen, die ihre Fähigkeiten, ihren Wissensstand und ihre Motivation nachhaltig beeinflussen. Deshalb arbeiten sie häufig weit unter ihrem Potenzial (Office for Standards in Education, 1996). Besonders betroffen sind sie, wenn sie von Schule und Lehrpersonen nicht unterstützt werden.

Weil sich Underachievement hauptsächlich in unterrichtlichen Settings manifestiert, gilt den Schulfaktoren besondere Beachtung. Entsprechend aufschlussreich sind die Befunde der Studien von Reis (2003) oder Baker, Bridger und Evans (1998), in denen aufgezeigt wird, dass ein inadäquates Curriculum und eine wenig herausfordernde Unterrichtsorganisation zur Entstehung von Langeweile beitragen und zusammen mit ungünstigen Interaktionsstrategien der Lehrperson und ebensolchen Einstellungen der Schülerinnen und Schüler (Butler-Por, 1993) massgeblich am Entstehen von Underachievement beteiligt sind. Einen wesentlichen Einfluss dürfte auch die Peergroup haben (Clasen \& Clasen, 1995). Solche Befunde basieren jedoch fast durchgehend auf Querschnittsvergleichen. Im Längsschnitt sind die Unterschiede zu Achievern möglicherweise geringer. Laut McCall, Evahn und Kratzer (1992) oder Hanses und Rost (1998) sind es vor allem die negativen Schul- und motivationalen Arbeitseinstellungen, welche Underachiever von Achievern unterscheiden.

In diesem Zusammenhang sind einige Studien von besonderem Interesse, die auf einen Zusammenhang von schulabsentem Verhalten und Underachievement hinweisen. Bei Seeley (1993) oder Renzulli und Park (2002) beispielsweise finden sich empirisch begründete Hinweise auf überdurchschnittlich begabte Un- 
derachiever, die teilweise massive Schulschwänzer waren und nicht selten zu Aussteigern und vorzeitigen Schulabbrechern wurden. Auch in einer Teilstudie Schweizer Längsschnittuntersuchung «Frühlesen und Frührechnen als soziale Tatsachen» konnten solche Befunde bestätigt werden (Stamm, 2005c). Gefunden wurden zwei Absentismusprofile: die so genannten «Blaumacher» als Jugendliche mit hoher intellektueller Kapazität und guten Schulleistungen, welche die Schule vornehmlich aus Unterforderungsgründen schwänzten und keine weiteren Probleme aufwiesen und die «Distanzierten», welche über die höchsten Intelligenzwerte verfügten, gleichzeitig jedoch unterdurchschnittliche Schulleistungen erzielten und deutliche Abgrenzungstendenzen gegenüber der Schule zeigten. Somit entspricht diese zweite Gruppe weitgehend einer der operationalen Definitionen von Underachievement.

Aus der Geschlechterperspektive betrachtet gilt das Konstrukt des Underachievements als männliches Phänomen (Colangelo et al., 1993; Flammer \& Keller, 1978; Heckhausen, 1980). Die zahlreich vorliegenden Belege berichten durchgehend von einem Verhältnis von Jungen zu Mädchen von 2:1 oder gar 3:1 (Colangelo et al., 1993; McCall, Evahn \& Kratzer, 2000; Peterson \& Colangelo, 1996; Reis \& McCoach, 2000). Jones und Myhill (2004) oder Hyde, Fennema und Lamon (1990) interpretieren die Ursache dieser Diskrepanz damit, dass Lehrpersonen aufgrund ihrer vorwiegend geschlechtstypischen Erwartungen den Jungen höhere kognitive Fähigkeiten und den Mädchen eine grössere Arbeitsverpflichtung zuschreiben und in erster Linie die Jungen mit Minderleistung assoziieren, minderleistende Mädchen hingegen weit seltener identifizieren. Unstrittig ist ebenfalls, dass der familiäre Kontext einen wichtigen Einflussfaktor darstellt. Die Forschungen von Benbow (1992), Freeman (1993), Ganzach (2000) oder Stamm (2005b) zeigen einen Interaktionseffekt zwischen den Genderstereotypen der Eltern und den Selbstwahrnehmungen, welche die Leistung beeinflussen. So haben Eltern gegenüber ihren überdurchschnittlich begabten Töchtern deutlich niedrigere und häufig der traditionellen Frauenrolle entsprechende Erwartungshaltungen, welche indirekt das Verhalten der Mädchen beeinflussen dürften.

Insgesamt zeichnen die hier berichteten Befunde ein eher negatives Bild überdurchschnittlich begabter Underachiever. Auf dieser Grundlage geht die hier präsentierte Untersuchung einer dreiteiligen Fragestellung nach:

(1)Unterscheiden sich die überdurchschnittlich begabten Underachiever und die überdurchschnittlich begabten Achiever der in leistungsbezogenen und beziehungsorientierten Einstellungs- und Verhaltensmustern?

(2)Gibt es Unterschiede zwischen den beiden Gruppen in Bezug auf Geschlecht, soziale Herkunft und Schulgrösse sowie Schultyp?

(3) Welche der untersuchten Faktoren sind am besten in der Lage, die Zugehörigkeit zur Gruppe der Underachiever resp. der Achiever vorauszusagen? 


\section{Methode}

\section{Stichprobe}

Die Studie bezieht sich auf die Stichproben zweier Schweizer Längsschnittstudien. Während das Projekt «Hoch begabt und «nur' Lehrling?» («HBL») die Exzellenzentwicklung überdurchschnittlich begabter Lehrlinge während der Berufsausbildung untersucht, fragt die Studie "Frühlesen und Frührechnen als soziale Tatsachen» («FLR») nach den Wirkungen des vorschulischen Kompetenzerwerbs auf die Leistungsentwicklung im Erwachsenenalter. Beide Projekte werden von der KTI-Berufsbildungsforschung, der beteiligten Deutschweizer Kantone und vom Fürstentum Liechtenstein finanziert. Das HBL-Projekt umfasst 376 Personen, das FLR-Projekt 360 Personen. Für die hier präsentierte Teilstudie herausgefiltert wurden alle Jugendlichen, welche in einem kognitiven Fähigkeitstest einen IQ-Prozentrang von $\geq 90$ erreichten. Im FLR-Projekt waren es der CFT 1 (Weiss \& Osterland, 1980) und der CFT 20 (Weiss, 1987), im HBLProjekt das Leistungsprüfungssystem L-P-S (Horn, 1983) und der T-dV2, der Test für zwei-dimensionale räumliche Vorstellungen von Skawran (1965). Im HBL-Projekt konnten auf diese Weise 180 Personen, im FLR-Projekt 84 Personen selektioniert werden. Die Zusammenführung dieser beiden Stichproben lässt sich aus vier Gründen legitimieren: Erstens haben die Probandinnen und Probanden den gleichen Jahrgang (1988). Zweitens stammen sie aus vergleichsweise ähnlichen Schulniveaus (zwischen 30\% und 35\% oberstes, $45 \%$ und 55\% mittleres und 20\% resp. 25\% unterstes Anforderungsniveau). Drittens liegen für beide Tests Untersuchungen vor, welche Korrelationen mit anderen Intelligenzund Schulreifetests im mittleren bis hohen Bereich nachweisen. Viertens sind die Instrumente zur Erfassung der Leistungs-, Schul- und Personaldaten sowie der Zeitpunkt der Befragung sehr ähnlich. Die FLR-Befragung fand im Herbst/Winter 2003/2004 statt, also kurz vor Ende der obligatorischen Schulzeit, die HBL-Befragung im August 2004, unmittelbar nach Beginn des ersten Lehrjahres. Die Fragen bezogen sich hier jedoch ausschliesslich auf die obligatorische Schulzeit. Ein Unterschied zwischen den beiden Befragungen war, dass die Untersuchung im FLR-Projekt ausschliesslich während des Unterrichts stattfand, im HBL-Projekt jedoch nur die Fähigkeitstests im Klassenverband durchgeführt wurden, während die Personaldaten schriftlich privat erhoben wurden. Die Schuldaten wurden in beiden Fällen über die Schulleitungen erfasst.

Auf der Grundlage dieser Stichrobe, die insgesamt 264 Personen umfasste, wurde anhand der nachfolgenden Selektionskriterien bestimmt, wer der Subpopulation der Achiever und wer der Subpopulation der Underachiever zugeteilt werden sollte.

(a) Überdurchschnittlich begabte Underachiever: IQ-Prozentrang $\geq 90^{1}$ und Schulleistungsprozentrang (Deutsch/Mathematik) $\leq 50$ :

Projekt FLR: n=25; Durchschnittsnote Deutsch/Mathematik 2.54 (min.=4, $\max .=1) ; s=0.45$; IQ: 126.26 . 
Projekt HBL: $\mathrm{n}=26$; Durchschnittsnote Deutsch/Mathematik 2.45 ( $\mathrm{min} .=4$, max.=1); s=0.65; IQ: 122.26 .

(b) Überdurchschnittlich begabte Achiever: IQ-Prozentrang $\geq 90$ und Schulleistungsprozentrang (Deutsch/Mathematik) $\geq 75$ :

Projekt FLR: n=59; Durchschnittsnote Deutsch/Mathematik 2.04; (min.=4, max.=1) s=0.34; IQ: 124.03 .

Projekt HBL: $\mathrm{n}=154$; Durchschnittsnote Deutsch/Mathematik 1.99; (min.=4, $\max .=1) \mathrm{s}=0.44 ; \mathrm{IQ}: 121.01$.

\section{Erhebungsinstrumente}

Die Erfassung der sozio-ökonomischen Hintergrundsvariablen inkl. Geschlecht und Schultyp erfolgte mittels Einzelfragen. Die Schulgrösse wurde anhand der vorliegenden Schuldaten bestimmt und sechs verschiedenen Typen zugeordnet. Zur Erfassung der leistungsbezogenen und beziehungsorientierten Einstellungsund Verhaltensmuster wurden die fünf in Tabelle 1 dargestellten Skalen verwendet.

Tabelle 1: Eingesetzte Skalen mit Beispielitems und interner Konsistenz

\begin{tabular}{|l|l|l|c|c|c|}
\hline \multicolumn{1}{|c|}{ Konstrukt } & \multicolumn{1}{|c|}{ Quelle } & \multicolumn{1}{|c|}{ Beispielitem } & $\begin{array}{c}\text { Item- } \\
\text { anzahl }\end{array}$ & $\begin{array}{c}\text { FLR } \\
\boldsymbol{\alpha}\end{array}$ & $\begin{array}{c}\text { HBL } \\
\boldsymbol{\alpha}\end{array}$ \\
\hline Schulschwänzen & $\begin{array}{l}\text { Schreiber-Kittl } \\
\text { \& Schröpfer } \\
(2002)\end{array}$ & $\begin{array}{l}\text { Ich habe die Schule geschwänzt, } \\
\text { weil der Unterricht langweilig } \\
\text { war. }\end{array}$ & 6 & .71 & .68 \\
\hline $\begin{array}{l}\text { Schulzufriedenheit } \\
\text { allgemein }\end{array}$ & $\begin{array}{l}\text { PISA Fragebo- } \\
\text { gen 2003 } \\
\text { (Q 27)* }\end{array}$ & $\begin{array}{l}\text { Vieles, was ich in der Schule } \\
\text { lernen sollte, war nutzlos. }\end{array}$ & 6 & .78 & .87 \\
\hline $\begin{array}{l}\text { Beziehungen zu } \\
\text { den Lehrpersonen }\end{array}$ & $\begin{array}{l}\text { PISA Fragebo- } \\
\text { gen 2003 } \\
\text { (Q 26)* }\end{array}$ & $\begin{array}{l}\text { Ich bin mit den meisten Lehr- } \\
\text { kräften gut ausgekommen. }\end{array}$ & 5 & .82 & .80 \\
\hline $\begin{array}{l}\text { Karrierepläne und } \\
\text { Zukunftsvorstell- } \\
\text { ungen }\end{array}$ & $\begin{array}{l}\text { Eigenentwick- } \\
\text { lung auf der } \\
\text { Grundlage von } \\
\text { Stein (2004) }\end{array}$ & $\begin{array}{l}\text { Ich möchte ein Studium } \\
\text { absolvieren (FH oder Uni). }\end{array}$ & 5 & .68 & .67 \\
\hline $\begin{array}{l}\text { Leistungsmotiva- } \\
\text { tion und Selbstre- } \\
\text { gulation }\end{array}$ & $\begin{array}{l}\text { Skala nach Wild } \\
\text { und Remy } \\
\text { (2002) }\end{array}$ & $\begin{array}{l}\text { Ich kann mich für die Erledi- } \\
\text { gung der Hausaufgaben gut } \\
\text { motivieren }\end{array}$ & 4 & 0.81 & 0.87 \\
\hline
\end{tabular}

Anmerkung: Der Range der Antwortskalen liegt zwischen 1 [trifft nicht zu] und 4 [trifft genau zu].

* Betrifft den in der Schweiz und im Fürstentum Liechtenstein verwendeten Fragebogen.

Die Skala Schulschwänzen misst die Variabilität und Intensität schulabsenten Verhaltens. Schulzufriedenheit und Lehrerbeziehungen sind zwei Skalen, um das Ausmass der persönlichen Zufriedenheit mit Schule und Unterricht sowie mit der Interaktion zu den Lehrkräften feststellen zu können. Die Karrierepläne geben Auskunft über das Ausmass an zielgerichteter beruflicher Zukunftsorientie- 
rung, und die Skala Leistungsmotivation/Selbstregulation, welche Wünsche und Absichten umschreibt, Erfolge zu erzielen und Misserfolge zu vermeiden und sich dabei in einem stabilen Zustand zu halten. Trotz der teilweise geringen Anzahl an Items, die in eine Skala eingegangen waren, sind die internen Konsistenzen zufrieden stellend bis gut.

\section{Auswertung}

Für die verwendeten Skalen wurden als erstes t-Tests gerechnet. Um den $\alpha$-Fehler zu korrigieren wurde der Bonferroni-Test verwendet und das $\alpha$ auf $\mathrm{p} \leq .01$ festgesetzt. Die Differenzen zwischen den Items Geschlecht, soziale Herkunft, Schulgrösse sowie Schultyp wurden mittels Chi-Quadrat-Analysen berechnet. Die Unterschiede zwischen den beiden Gruppen wurden mittels univariater Varianzanalysen in den Skalen Schulschwänzen, Schulzufriedenheit, Lehrerbeziehungen und Karrierepläne geprüft. Anschliessend wurden Regressionsanalysen durchgeführt um zu eruieren, welche der vier Faktoren (als Prädiktoren) am ehesten Underachievement voraussagen können. Zur Kennzeichnung praktischer Signifikanz wurde das Effektstärkenmass d verwendet. Gemäss den Richtlinien von Cohen (1988) bezeichnet ein d-Wert um 0.2 einen kleinen, um 0.5 einen mittleren, um 0.8 einem grossen und um 1.30 einem sehr grossen Effekt.

\section{Ergebnisse}

Unterschiede in den Einstellungs- und Verhaltensmustern Aus Tabelle 2 werden die Ergebnisse der t-Tests ersichtlich. Die Mittelwertsunterschiede zwischen den Einschätzungen der beiden Leistungsgruppen in Bezug auf das Schulschwänzen, die Lehrerbeziehungen und die Leistungsmotivation sind alle statistisch signifikant. Die Effektstärken sind gross, sie reichen von .55 (Leistungsmotivation) bis zu 1.08 (Schulschwänzen). Nicht signifikant sind hingegen die Mittelwertsdifferenzen in Bezug auf die allgemeine Schulzufriedenheit und die Karrierepläne. Sowohl Underachiever als auch Achiever zeigen aus der Rückschau nicht nur eine recht hohe Schulzufriedenheit (Mittelwerte 2.98 res. 3.07) sondern auch gezielte Zukunftspläne (Mittelwerte 3.10 resp. 3.21). Die Effektstärken sind mit .22 und .28 klein. 
Tabelle 2: Mittelwertsunterschiede in den verwendeten Skalen

\begin{tabular}{|lcccccc|}
\hline Skalen & \multicolumn{7}{c}{ Achiever $(\mathrm{n}=213)$} & Underachiever $(\mathrm{n}=51)$ & \multirow{2}{*}{$\mathrm{d}$} & $\mathrm{d}$ \\
& $\mathrm{M}$ & $\mathrm{s}$ & $\mathrm{M}$ & $\mathrm{s}$ & & \\
\hline Schulschwänzen & 2.34 & .57 & 2.74 & .89 & .001 & 1.08 \\
Schulzufriedenheit allgemein & 3.07 & .64 & 2.98 & 1.02 & .09 & .22 \\
Beziehungen zu den Lehrpersonen & 2.72 & .45 & 2.21 & .95 & .04 & .81 \\
Karrierepläne/Zukunftsvorstellungen & 3.21 & .44 & 3.10 & .70 & .12 & .28 \\
Leistungsmotivation und Selbstregulation & 2.98 & .87 & 2.03 & 1.22 & .02 & .55 \\
\hline
\end{tabular}

\section{Unterschiede in Bezug auf Geschlecht, soziale Herkunft, Schulgrösse und Schultyp}

Von Interesse ist als nächstes, ob es Geschlechtsunterschiede zwischen Achievern und Underachievern gibt, ob sie sich hinsichtlich ihres kulturellen und sozialen Hintergrundes unterscheiden und inwiefern sich Differenzen in Schulgrösse und Schultyp abzeichnen. Aus Tabelle 3 wird ersichtlich, dass es zwischen den beiden Gruppen signifikante Geschlechtsunterschiede gibt. So stehen $50.5 \%$ männlichen Achievern $(n=111)$ 49.5\% weibliche Achiever $(n=102)$ gegenüber, während es bei den Underachievern 70.7\% Jungen ( $n=36)$ und 29.3\% Mädchen $(\mathrm{n}=15)$ sind. Damit lassen auch unsere Ergebnisse zu, Underachievement als vorwiegend männliches Phänomen zu beschreiben. Mit Blick auf die Nationalität ergeben sich hingegen keine relevanten Unterschiede, obwohl in der Underachievergruppe mehr Jugendliche ausländischer Herkunft vertreten sind als in der Achievergruppe (10.7\% zu 7.4\%). Anders sieht es wiederum mit Blick auf die soziale Herkunft aus. Underachiever kommen tendenziell aus bildungsnäheren Elternhäusern als Achiever. Während 51.1\% der Väter von Underachievern über einen Abschluss einer Höheren Fachschule, einer Fachhochschule oder Universität verfügt, sind es bei den Achievern lediglich 37.9\%. Unsere Chi-QuadratAnalysen zeigen ferner aufschlussreiche Ergebnisse im Hinblick auf die Grösse der besuchten Schulen. Während Underachiever bevorzugt aus mittelgrossen Schulen von 200 bis 600 Schülern stammen (65.6\%), sind Achiever eher in kleinen und kleineren Schulen bis zu 200 Schülern unterrichtet worden (52.5\%). Diese signifikanten Unterschiede zwischen den beiden Leistungsgruppen lassen den Schluss zu, dass die Grösse der Schule keine unbedeutende Rolle spielt. Zumindest scheint die Tatsache interpretationsbedürftig, dass es in grossen Schulen wenig Underachiever, aber relativ viele Achiever in kleinen Schulen gibt. Gleiches gilt für die Differenzierung nach Schultyp: Denn die Tatsache, dass deutlich mehr Achiever als Underachiever (88.2\% zu 75.2\%) öffentliche Schulen besuchten, verweist darauf, dass sich Privatschulen deutlich häufiger mit Underachievern auseinandersetzen müssen. Möglicherweise stellten Minderleistungen einen Grund für den Wechsel in eine Privatschule dar. 
Tabelle 3: Demografische Charakteristika von überdurchschnittlich begabten Underachievern und überdurchschnittlich begabten Achievern

\begin{tabular}{|c|c|c|c|c|}
\hline Merkmal & & $\begin{array}{l}\text { Underachiever } \\
\qquad(\mathrm{n}=51)\end{array}$ & $\begin{array}{c}\text { Achiever } \\
(\mathrm{n}=213)\end{array}$ & $x^{2}$ \\
\hline \multirow{2}{*}{ Geschlecht } & männlich & 70.7 & 50.5 & \\
\hline & weiblich & 29.3 & 49.5 & $6.866^{*}$ \\
\hline \multirow{2}{*}{ Nationalität } & Schweiz & 89.3 & 92.6 & \\
\hline & andere & 10.7 & 7.4 & 0.664 \\
\hline \multirow{6}{*}{$\begin{array}{l}\text { Soziale Her- } \\
\text { kunft } \\
\text { (Berufsab- } \\
\text { schluss Vater) }\end{array}$} & Obligatorische Schule & 7.0 & 9.3 & \\
\hline & Anlehre & 8.9 & 11.3 & \\
\hline & Berufslehre & 13.8 & 16.3 & \\
\hline & Diplom/Meister & 19.2 & 25.2 & \\
\hline & Höhere Fachschule & 26.1 & 16.8 & \\
\hline & Fachhochschule/Universität/ETH & 25.0 & 21.1 & $16.427^{*}$ \\
\hline \multirow{6}{*}{ Schulgrösse } & $<30$ & 0 & 0 & \\
\hline & $31-100$ & 9.7 & 20.2 & \\
\hline & $101-200$ & 17.2 & 32.3 & \\
\hline & $201-400$ & 40.3 & 23.1 & \\
\hline & $401-600$ & 25.3 & 28.6 & \\
\hline & $601-900$ & 7.5 & 4.8 & $14.453^{*}$ \\
\hline \multirow{2}{*}{ Schultyp } & öffentliche Schule & 75.2 & 88.2 & \\
\hline & Privatschule & 24.8 & 11.8 & $6.716^{*}$ \\
\hline
\end{tabular}

${ }^{*} \mathrm{p}<.05 ;{ }^{* *} \mathrm{p}<.01 ;{ }^{* * *} \mathrm{p}<.00$

Als nächstes wurde eine logistische Regressionsanalyse durchgeführt. Sie bietet die Möglichkeit, den nicht-linearen Einfluss mehrerer unabhängiger Variablen (Risikofaktoren) auf eine dichotome Zielgrösse zu untersuchen und zu bestimmen, welche Modellfaktoren am besten in der Lage sind, die Gruppenzugehörigkeit eines Schülers oder einer Schülerin zu bestimmen. Unser Modell mit den fünf Faktoren klassierte insgesamt 89.1\% Probandinnen und Probanden entweder als Underachiever oder als Achiever. Die fehlenden 11.1\% können aufgrund der Missings bei den Prädiktorvariablen nicht klassiert werden. Die Regressionsanalyse wurde stufenweise durchgeführt. In einem ersten Schritt wurden alle fünf Prädiktoren für die Vorhersage von Achievement/Underachievement beigezogen. Tabelle 4 gibt Auskunft über die Befunde. Aufgrund der Multikollinearität zwischen den fünf Faktoren deckt der Wald-Test auf, dass nur zwei der fünf Faktoren (Schulschwänzen und Lehrerbeziehungen) signifikante Prädiktoren darstellen und somit die Leistungsfähigkeit voraussagen können. Dies trifft weder für die Schulzufriedenheit noch für die Karrierepläne oder die Leistungsmotivation $\mathrm{zu}(\mathrm{p}>$.10). 
Tabelle 4: Ergebnisse der logistischen Regressionsanalyse mit fünf Faktoren

\begin{tabular}{|lccccccc|}
\hline Prädiktorvariable & b & SD & Wald & df & Sig & R & $\operatorname{Exp}(\mathbf{b})$ \\
\hline Schulschwänzen & .65 & .28 & .6 .54 & 1 & .01 & .15 & 1.98 \\
\hline Schulzufriedenheit & -.63 & .25 & .008 & 1 & .61 & .00 & .95 \\
\hline Beziehungen zu Lehrpersonen & .76 & .30 & 6.75 & 1 & .01 & .14 & 2.12 \\
\hline Karrierepläne & .05 & .24 & .007 & 1 & .96 & .00 & 1.01 \\
\hline $\begin{array}{l}\text { Leistungsmotivation } \\
\text { Arbeitsverpflichtung }\end{array}$ & .25 & .22 & 1.74 & 1 & .17 & .01 & 2.12 \\
\hline
\end{tabular}

Anmerkungen:

$\mathrm{N}=234 ;{ }^{*}: \mathrm{p}<.05 ;{ }^{* *}:<.01 ; * * *$ : $<.001$

$\chi^{2}(5)$ Gesamtmodell $=65.5$ ( $\left.\mathrm{p}>.10\right) ; \chi^{2}$ als Differenz zwischen der -2 Log-Likelihood des o-Modells und der -2 Log-Likelihhod der Modellschätzung.

Deshalb wurde in einem weiteren Schritt ein zweites Modell mit den unabhängigen Variablen Schulschwänzen und Lehrerbeziehungen gerechnet. Es findet sich ein signifikantes $\chi^{2}(2)=54.2, p<.01$, was anzeigt, dass die Koeffizienzwerte im Modell statistisch signifikant von 0 verschieden sind (Cox und Snell $\mathrm{R}^{2}=.318$; Negelkerke=.421) $)^{2}$. Diese Ergebnisse verweisen darauf, dass das Modell mit lediglich zwei Faktoren signifikant besser in der Lage ist, Schülerinnen und Schüler als Underachiever oder Achiever zu klassieren als das Modell mit den fünf Faktoren.

Die nachfolgenden Analysen gehen der Frage nach, inwieweit demografische und sozialstrukturelle Merkmale die Ausprägung von Underachievement beeinflussen können. $\mathrm{Zu}$ diesem Zweck wurde eine weitere logistische Regression gerechnet, wobei die beiden unabhängigen Variablen Schulschwänzen und Lehrerbeziehungen erst am Schluss in die Regressionsgleichung eingeführt wurden. Die Antwortkategorien von Schulschwänzen und Lehrerbeziehungen gingen dabei als Dummy-Variablen in die logistische Regression ein, indem "Ich habe bisher nie geschwänzt» respektive «Die Beziehungen zu den Lehrpersonen sind miserabel» mit «0» kodiert wurde und alle übrigen Angaben mit «1». Durch diese Vorgehensweise wird der Beitrag sichtbar, den die beiden Variablen nach Kontrolle der demografischen Merkmale für das Leistungsverhalten haben. Die Tabellen 5 und 6 zeigen die Ergebnisse. Aus der Tabelle 5 wird ersichtlich, dass der Vorhersagebeitrag von Schulschwänzen mit $\Delta \chi^{2}(4)=305.51 \quad(\mathrm{p}<.01)$ immer noch signifikant ist. Gleiches gilt für die Befunde in Tabelle 6 mit $\Delta \chi^{2}(4)=$ 310.55 ( $\mathrm{p}<.01)$ für Lehrerbeziehungen. Somit leisten Schulschwänzen und ungünstige Lehrerbeziehungen auch nach Kontrolle der sozial-strukturellen Variablen einen signifikanten Vorhersagebeitrag für Underachievement. 
Tabelle 5: Logistische Regression von Schulschwänzen auf Underachievement/Achievement unter Kontrolle demografischer Merkmale

\begin{tabular}{|lccccccc|}
\hline Prädiktorvariable (UV) & b & SE & Wald & df & Sig & R & Exp(b) \\
\hline Geschlecht & & & & & & & \\
(0: weiblich) & .284 & .051 & 30.80 & 1 & .07 & .14 & 1.172 \\
\hline Bildungsstatus der Eltern &. .004 & .002 & 2.564 & 5 & .13 & .00 & \\
\hline $\begin{array}{l}\text { (0=FH/Universität) } \\
\quad \text { Obligatorische Schule }\end{array}$ & .169 & .081 & 4.378 & 1 & .23 & .15 & 1.185 \\
$\quad$ Anlehre & .169 & .064 & 6.934 & 1 & .17 & .22 & 1.184 \\
$\quad$ Berufslehre & .317 & .088 & 12.847 & 1 & .12 & .15 & 1.373 \\
$\quad$ Diplom/Meister & .213 & .051 & 7.132 & 1 & .15 & .04 & 1.997 \\
$\quad$ Höhere Fachschule & .308 & .056 & 8.123 & 1 & .06 & .23 & 1.543 \\
\hline Schulgrösse & & & 13.564 & 5 & .03 & .12 & \\
(0: $<30)$ & & & & & & & \\
\hline 31-100 &. .335 & .134 & 6.297 & 1 & .16 & .21 & .715 \\
$\quad$ 101-200 & .231 & .061 & 3.182 & 1 & .05 & .31 & 1.112 \\
$\quad$ 201-400 & .417 & .051 & 7.123 & 1 & .20 & .02 & 1.345 \\
$\quad$ 401-600 & .312 & .035 & 15.212 & 1 & .06 & .03 & 1.523 \\
$\quad$ 601-900 & .153 & .205 & 3.555 & 1 & .11 & .01 & 1.670 \\
\hline Schultyp & .119 & .034 & 12.343 & 1 & .02 & .32 & .996 \\
(0: Privatschule) & & & & & & & \\
\hline Schulschwänzen (letzte 6 Mon.) & & & & 4 & .001 & .09 & \\
(0: nein) & & & & & & & \\
\hline \multicolumn{1}{l}{ 1 Tag } & .597 & .065 & 71.86 & 1 & .02 & .02 & 11.818 \\
2-4 Tage & 1.012 & .070 & 207.99 & 1 & .001 & .00 & 12.756 \\
5-10 Tage & 1.142 & .104 & 163.90 & 1 & .001 & .00 & 31.087 \\
$\quad$ Mehr als 10 Tage & 1.452 & .179 & 183.00 & 1 & .02 & .00 & 42.165 \\
Konstante & -2.165 & .170 & 210.770 & 1 & & & \\
\hline
\end{tabular}

Anmerkungen:

$\mathrm{N}=264 ;^{*}: \mathrm{p}<.05 ;{ }^{* *}:<.01 ;{ }^{* * *}: \mathrm{p}<.001$

$\chi^{2}(14)$ Gesamtmodell $=789.79(\mathrm{p}<.01)$

$\Delta \chi^{2}(4)$ für Schwänzen $=305.51(\mathrm{p}<.01)$ 
Tabelle 6: Logistische Regression von Lehrerbeziehungen auf Underachievement/Achievement unter Kontrolle demografischer Merkmale

\begin{tabular}{|c|c|c|c|c|c|c|c|}
\hline Prädiktorvariable (UV) & b & SE & Wald & df & Sig & $\mathrm{R}$ & $\operatorname{Exp}(b)$ \\
\hline Geschlecht & 284 & .051 & 30.80 & 1 & 0.11 & .34 & 1.328 \\
\hline (0: weiblich) & & & \multicolumn{5}{|c|}{.07} \\
\hline Bildungsstatus der Eltern & & & & 5 & .13 & .00 & \\
\hline \multicolumn{8}{|l|}{ (0=FH/Universität) } \\
\hline Obligatorische Schule & .169 & .081 & 4.378 & 1 & .33 & .33 & 1.126 \\
\hline Anlehre & .169 & .034 & 7.004 & 1 & .15 & .42 & 1.453 \\
\hline Berufslehre & .320 & .098 & 10.844 & 1 & .22 & .02 & 1.473 \\
\hline Diplom/Meister & .113 & .051 & 6.002 & 1 & .001 & .03 & .907 \\
\hline Höhere Fachschule & .300 & .023 & 6.123 & 1 & .06 & .00 & .990 \\
\hline Schulgrösse & & & & 5 & .03 & .09 & \\
\hline \multicolumn{8}{|l|}{$(0:<30)$} \\
\hline $31-100$ & -.110 & .169 & .424 & 1 & .36 & & .715 \\
\hline $101-200$ & .245 & .061 & 3.182 & 1 & .15 & .33 & 1.06 \\
\hline $201-400$ & .417 & .051 & 7.123 & 1 & .02 & .23 & 1.345 \\
\hline $401-600$ & .312 & .035 & 15.212 & 1 & .06 & .04 & 1.523 \\
\hline $601-900$ & .153 & .205 & 3.555 & 1 & .15 & .02 & 1.670 \\
\hline Schultyp & .119 & .034 & 12.343 & 1 & .01 & .23 & 1.675 \\
\hline \multicolumn{8}{|l|}{ (0: Privatschule) } \\
\hline Beziehungen zu Lehrpersonen & 0.24 & 0.27 & 1.75 & 4 & .05 & .21 & 1.24 \\
\hline \multicolumn{8}{|l|}{ (0: miserabel) } \\
\hline ziemlich schlecht & .417 & .078 & 745.923 & 1 & .02 & .02 & 1.517 \\
\hline mittel & .561 & .142 & 34.001 & 1 & .011 & .03 & 1.802 \\
\hline ziemlich gut & .432 & .145 & 12.832 & 1 & .07 & .12 & 1.702 \\
\hline sehr gut & .560 & .101 & 34.221 & 1 & .03 & .08 & 1.675 \\
\hline Konstante & -2.537 & .172 & 216.22 & 1 & & & \\
\hline
\end{tabular}

Anmerkungen:

$\chi^{2}(14)$ Gesamtmodell $=906.65(\mathrm{p}<.01)$

$\Delta \chi^{2}(4)$ für Lehrerbeziehungen $=374.55(\mathrm{p}<.01)$

\section{Diskussion}

Der Zweck dieser Studie lag darin, die rätselhafte Gruppe der überdurchschnittlich begabten Underachiever im Hinblick auf einige sozial-strukturelle und personbezogene Merkmale zu untersuchen und Einsicht in ihre Charakteristika zu erlangen. $\mathrm{Zu}$ diesem Zweck wurde sie mit einer Gruppe überdurchschnittlich begabter Achiever verglichen. Im Ergebnis lässt sich die empirische Evidenz unserer Befunde in dreierlei Hinsicht charakterisieren:

1. Die Ergebnisse streichen eine klare Differenz zwischen den beiden untersuchten Gruppen hervor. Underachiever sind in erster Linie männlichen Ge- 
schlechts und stammen aus eher bildungsnahen Familien. Sie besuchen bevorzugt grössere und private Schulen, was nahe legt, die Thematik auch aus einer institutionellen Perspektive zu betrachten.

2. Mit Bezug auf die personalen Merkmale scheint nicht unerwartet, dass Underachiever deutlich weniger leistungsmotiviert sind als Achiever und grössere Probleme mit der Selbstregulation haben. Dieser durch zahlreiche Forschungsbefunde replizierten Tatsache steht der erstaunliche Befund gegenüber, wonach sich die beiden untersuchten Gruppen weder in ihren Schuleinstellungen noch in ihren Karriereplänen unterscheiden. Underachiever zeigen keine ausgeprägten schulfeindlichen Haltungen oder eine «no-future»-Einstellung, wie sie teilweise in der populärwissenschaftlichen Literatur immer wieder skizziert werden.

3. Im Gegensatz dazu - und mit den gängigen Vorstellungen kompatibel - sind die Befunde zu den Interaktionsmustern mit Lehrkräften. Sie lassen vermuten, dass Schullaufbahnen von Underachievern durch vorwiegend negative Lehrerbeziehungen gekennzeichnet sind, in denen sich das Ausmass ihrer Frustration widerspiegelt. Damit einher geht die Tatsache, dass Underachiever auch nach Kontrolle der sozial-strukturellen Merkmale, deutlich seltener zu ihren Lehrpersonen gute Beziehungen pflegen als dies für die Achiever zutrifft und überzufällig häufiger Schulschwänzer sind. Zu 45\% betreiben sie dies in einem Ausmass, das bereits Formen der Regelmässigkeit annimmt. Unsere Daten weisen darauf hin, dass Underachievement-Karrieren durch Schulschwänzen weiter provoziert werden können und Gleiches für schlechte Lehrerbeziehungen gilt.

Insgesamt legen diese Befunde nahe, das Augenmerk verstärkt auf Underachiever zu richten und dabei sowohl ihr Absentismusverhalten als auch die Beziehungsstrukturen zu ibren Lehrpersonen in den Blick zu nehmen. Wenn überdurchschnittlich begabte Jugendliche mit wenig aversiven Schuleinstellungen trotzdem von der Schule driften und möglicherweise ungünstige Lehrer-Schüler-Interaktionsstrukturen einer der Gründe für ihre Schulabsenz darstellen, dann lässt dieser Befund die Vermutung zu, dass Einflussgrössen des schulischen Feldes, insbesondere Beziehungsmuster und eventuell auch Unterrichtsmerkmale, an ihrem Verhalten ursächlich beteiligt sein dürften. Auf der Folie einer der Eingangsfragen - inwiefern überdurchschnittliche Begabung und schulmeidende Verhaltensformen miteinander korrelieren - und der Erkenntnis, dass die Grösse einer Schule offenbar eine Rolle spielt, lenken solche schul- und unterrichtsbezogenen Einflussgrössen die Aufmerksamkeit auf die institutionelle Perspektive und damit auf die Schulqualität.

Da grosse Schulen offensichtlich in stärkerem Ausmass mit Underachievement, Schulschwänzen und negativen Lehrerbeziehungen konfrontiert werden, stellt sich die Frage, ob ihre Unterstützungs-, Interaktions- und Kontrollsysteme mit grösseren Schwierigkeiten verbunden sind als dies für kleine Schulen zutrifft. In Anlehnung an und Erweiterung von Holtappels (1995) Gedanken zum 
Zusammenhang von Schulkultur und Schulqualität könnte davon ausgegangen werden, dass in grossen Schulen mit einer hohen Underachiever-Quote förderorientierte und regelstrukturierte Schulklimabedingungen eher fehlen und sich dies deshalb sowohl auf Leistungsbereitschaft als auch auf Interaktionsstrukturen negativ und absenzverstärkend auswirkt. Damit laufen Schulen Gefahr, ein Umfeld zu erzeugen, das Werte, Einstellungen und Verhaltensweisen von Underachievern in die falsche Richtung beeinflusst und sich daraus negative Zirkelprozesse ergeben: Underachiever haben allgemein ein eher niedriges Prestige. Durch ihre eher negativen Verhaltensweisen motivieren sie ihre Lehrpersonen, sie zusätzlich wieder negativ zu belohnen. Möglich wäre aber auch, dass die verstärkte Orientierung an Schulvergleichstests der Lehrerschaft damit einhergeht, leistungsschwache Schülerinnen und Schüler - und damit auch Underachiever als Hindernis zu erachten, um gute Testergebnisse zu erzielen. Solche Zusammenhänge sind allerdings nicht zwingend aus den vorliegenden Daten abzulesen. Deshalb müssen an dieser Stelle auch einige Begrenzungen der Studie diskutiert werden.

Einschränkungen ergeben sich erstens mit Blick auf die Zeitpunkte der Befragung, die entweder kurz vor oder kurz nach dem Abschluss der obligatorischen Schulzeit stattfand und deshalb auf die aktuellen Einstellungsmuster der Underachiever insofern einen wesentlichen Einfluss gehabt haben dürfte, als sie ihren Wechsel in die nachfolgende Ausbildungsstufe entweder mit positiven Gefühlen «der zweiten Chance», möglicherweise aber auch mit Zukunftsängsten und -sorgen verbanden. Insofern können die hier vorgelegten Befunde lediglich explorativen Charakter haben. Zweitens ist zu beachten, dass Underachievement in dieser Untersuchung als abhängige Variable definiert wurde und damit die Richtung der Effekte vorgegeben war. Diese Frage ist jedoch in der Forschung bislang kaum geklärt, so dass offen bleiben muss, ob Persönlichkeitsmerkmale für das Entstehen von Minderleistung verantwortlich sind oder ob diese erst ungünstige Persönlichkeitsmerkmale zu Tage fördert. Unser Untersuchungsdesign orientiert sich allerdings an amerikanischen Studien von Callahan (1991), McCoach und Siegle (2003), Reis und McCoach (2000) sowie von Davis und Rimm (2004), die Underachievement als eine mögliche Antwort auf nicht adäquates Verhalten der Umwelt interpretieren, das wiederum die Persönlichkeitsentwicklung empfindlich stört. Schliesslich ist zu berücksichtigen, dass Underachiever in unseren Befunden durchgehend höhere Standardabweichungen aufweisen und deshalb davon auszugehen ist, dass sie die weit heterogenere Gruppe bilden als dies für Achiever zutrifft. Jede Interpretation unserer Befunde muss deshalb immer die Reflexion einschliessen, dass es möglicherweise viele differente Profile von Underachievern gibt, die mit weiteren, hier nicht diskutierten Faktoren einhergehen können.

Insgesamt lassen sich die Befunde zwar zu keinem Gesamtbild zusammenfügen, weil die komplexen und interdependenten Zusammenhänge zwischen den sozial-strukturellen und personalen Variablen sowie der institutionellen Perspek- 
tive in dieser Studie nicht untersucht wurden, doch verweisen sie mit einiger Sicherheit auf die Notwendigkeit, das Konstrukt des Underachievements in einen neuen Kontext zu stellen, indem seine vielfältigen Erscheinungsformen aus der Etikettierung als allein individuell verantwortetes Problem herausgelöst und um die institutionell-soziale Perspektive erweitert werden. Damit jedoch erscheint auch die Frage nach der "Humanverträglichkeit der Schule» in einem neuen Licht.

\section{Anmerkungen}

1 Es ist zu bemerken, dass der Prozentrang $\geq 90$ einem IQ von $\geq 120$ entspricht. Damit weicht dieses Kriterium vom häufig gesetzten Cut-off von 130 Punkten ab. Der Grund liegt darin, dass der Zweck der Identifikation in unseren beiden Projekten nicht in erster Linie in einem trennscharfen Testverfahren liegt, sondern in der Beschreibung und Erklärung der Entwicklungsverläufe von Kindern mit akzeleriertem Kompetenzerwerb resp. von Auszubildenden mit überdurchschnittlichen Begabungsprofilen. Die Messlatte wurde deshalb gesenkt, um eine Vielzahl überdurchschnittlich Begabter identifizieren zu können.

2 Diese Pseudo-R2-Masse sind ungefähr analog zum R2 in linearen Regressionen.

\section{Literatur}

Anastasi, A. (1976). Psychological testing. New York: Macmillan.

Baker, J. A., Bridger, R. \& Evans, K. (1998). Models of underachievement among gifted preadolescents: The role of personal, family, and school factors. Gifted Child Quarterly, 42, 514.

Baum, S., Renzulli, J. S. \& Hébert, T. P. (1995). The prism metaphor: A new paradigm for reversing underachievement. (Collaborative Research Study 95310). Storrs, CT: The National Research Center on the Gifted and Talented.

Benbow, C. P. (1992). Mathematical talent: Its origins and consequences. In N. Colangelo, S. G. Assouline \& D. L. Ambroson (Eds.), Talent development: Proceedings from the 1991 Henry B. and Jocelyn Wallace national research symposium on talent development (pp. 95123). Unionville, NY: Trillium Press.

Brown, B. B., Mounts, N., Lamborn, S. D. \& Steinberg, L. (1993). Parenting practices and peer group affiliation in adolescence. Child Development, 64, 467-482.

Butler-Por, N. (1993). Underachieving gifted students. In K. A. Heller, F. J. Mönks \& A. H. Passow (Eds.), International handbook of research and development of giftedness and talent (pp. 649-668). Oxford: Pergamon.

Callahan, C. M. (1991). An update on gifted females. Journal for the Education of the Gifted, 14, 284-312.

Clasen, D. R, \& Clasen, R. E. (1995). Underachievement of highly able students and the peer society. Gifted and Talented International, 10 (2), 67-75.

Cohen, J. (1988). Statistical power analysis for the behavioral sciences. Hillsdale, NJ: Lawrence Earlbaum Associates.

Colangelo, N., Kerr, B., Christensen, P. \& Maxey, J. (1993). A comparison of gifted underachievers and gifted high achievers. Gifted Child Quarterly, 37, 155-160.

Davis, G. A. \& Rimm, S. B. (2004). Education of the gifted and talented. Boston: Pearson Education.

Diaz, E. I. (1998). Perceived factors influencing the academic underachievement of talented students of Puerto Rican descent. Gifted Child Quarterly, 42, 105-122.

Dowdall, C. B. \& Colangelo, N. (1982). Underachieving gifted students: Review and implications. Gifted Child Quarterly, 26, 179-184. 
Durr, W. (1964). The gifted students. New York: Oxford University Press.

Flammer, A. \& Keller, B. (1978). Overachievement und Underachievement. In K. J. Klauer (Hrsg.), Handbuch der Pädagogischen Diagnostik, Band 2 (S. 1037-1044). Düsseldorf: Schwann.

Ford, D. Y. (1996). Reversing underachievement among gifted black students. New York: Teachers College Press.

Freeman, J. (1992). The development of infants. In F. J. Mönks \& A. M. Peters (Eds.), Talent for the future (pp. 23-36). Assen: Van Gorcum.

Freeman, J. (1993). Boredom, high ability and achievement. In V. P. Varma (Ed.), How and why children fail (pp. 29-40). London: Jessica Kingsley Publishers.

Ganzach, Y. (2000). «Parents» education, cognitive ability, educational expectations and educational attainment: Interactive effects. British Journal of Educational Psychology, 70, 419-441.

Hanses, P. \& Rost, D. (1998). Das "Drama» der hochbegabten Underachiever - "Gewöhnliche» oder «aussergewöhnliche» Underachiever? Zeitschrift für Pädagogische Psychologie, $12,53-71$.

Heckhausen, H. (Hrsg.). (1980). Fähigkeit und Motivation in erwartungswidriger Schulleistung. Göttingen: Hogrefe.

Heller, K. A., Mönks, F. \& Passow, H. A. (Eds.). (2000). International handbook of giftedness and talent. Amsterdam: Elsevier.

Holling, H. \& Preckel, F. (2005). Der BIS-HB - Diagnostik von Hochbegabung und allgemeinem sowie bereichsspezifischem schulischem Underachievement. Journal für Begabtenförderung, 1, 31-40.

Holtappels, H.-G. (1995). Ganztagserziehung als Gestaltungsrahmen der Schulkultur - Modelle und Perspektiven für ein zeitgemäßes Schulkonzept. In ders. (Hrsg.), Ganztagserziehung in der Schule (S. 12-48). Opladen: Leske + Budrich.

Horn, W. (1983). Horn, W. (1983). Das Leistungsprüfsystem (L-P-S.). Göttingen: Hogrefe.

Hyde, J. S, Fennema, E. \& Lamon, S. J. (1990). Gender differences in mathematics performance: A meta-analysis. Psychological Bulletin, 107, 139-155.

Jeon, K. W. \& Feldhusen, J. F. (1993). 'Teachers' and parents' perceptions of social-psychological factors of underachievement of the gifted in Korea and the United States. Gifted Education International, 9, 115-119.

Jones, S. \& Myhill, D. (2004). 'Troublesome boys' and 'compliant girls': gender identity and perceptions of achievement and underachievement. British Journal of Sociology of Education, 25, 547-561.

Klauer, K. J. (1990). Overachievement \& Underachievement revisited: Ein zwei-Fehler-kontrolliertes Modell zur Diagnostik erwartungswidriger Schulleistung. Diagnostica, 36, 299309.

McCall, R. B., Evahn, C. \& Kratzer, L. (1992). High school underachievers: What do they achieve as adults? Newbury Park: Sage.

McCoach, D. B. \& Siegle, D. (2003). Factors that differentiate underachieving students from achieving students. Gifted Child Quarterly, 47, 144-154.

Office for Standards in Education (OFSTED) (Ed.). (1996). Recent research on the achievements of ethnic minority pupils. London: Office for Standards in Education.

Peters, W. A. M., Grager-Loidl, H. \& Supplee, P. (2000). Underachievement in gifted children and adolescents. In K. A. Heller, F. J. Mönks, R. J. Sternberg \& R. F. Subotnik (Eds.), International handbook of giftedness and talent (pp. 609-620). Amsterdam: Elsevier.

Peterson, J. S. \& Colangelo, N. (1996). Gifted achievers and underachievers: A comparison of patterns found in school files. Journal of Counseling and Development, 74, 399-407.

Prause, G. (1996). Genies in der Schule. Reinbek: Rowohlt.

Raph, J. B., Goldberg, M. L. \& Passow, A. H. (1966). Bright underachievers. New York: Teachers College Press. 
Reis, S. M. \& McCoach, D. B. (2000). The underachievement of gifted students: What do we know and where do we go? Gifted Child Quarterly, 44, 152-170.

Reis, S. M. (2003). Reconsidering regular curriculum for high achieving students, gifted underachievers, and the relationship between gifted and regular education. In J. H. Borland (Ed.), Rethinking gifted education (pp. 186-200). New York: Teacher's College Press.

Reis, S. M., Hebert, T. P., Diaz, E. P., Maxfield, L. R. \& Ratley, M. E. (1995). Case studies of talented students who achieve and underachieve in an urban high school (Research Monograph 95120). Storrs, CT: University of Connecticut, National Research Center for the Gifted and Talented.

Renzulli, J. S. Park, S. (2002): Giftedness and high school dropouts: Personal, family, and schoolrelated factors. Storrs, CT: The National Research Center on the Gifted and Talented, University of Connecticut.

Richert, E. S. (1991). Patterns of underachievement among gifted students. In J. H. Borland (Ed.), Understanding the gifted adolescent (pp. 139-162). New York: Teachers College Press.

Rost, D. \& Hanses, P. (1997). Wer nichts leistet, ist nicht begabt? Zeitschrift für Entwicklungspsychologie und Pädagogische Psychologie, 29, 1, 167-177.

Schreiber-Kittel, M. \& Schröpfer, H. (2002). Abgeschrieben? Ergebnisse einer empirischen Untersuchung über Schulverweigerer. Opladen: Leske + Budrich.

Seeley, K. (1993). Gifted students at risk. In: Silverman, L. K. (Ed.), Counseling the gifted and talented (pp. 263-275). Denver: Love Publishing Company.

Shaw, M. C. \& McCuen, J. T. (1960). The onset of academic underachievement in bright children. Journal of Educational Psychology, 51, 103-108.

Skawran, W. (1965). Ein Test für zwei-dimensionale räumliche Vorstellungen. Diagnostica, $11,41-45$.

Smith, E. (2003). Understanding underachievement: an investigation into the differential attainment of secondary school pupils. British Journal of Sociology of Education, 24, 5, 574-586.

Sparfeldt, J. R. \& Schilling, S. (2006). Underachievement. In D. H. (Hrsg.), Handwörterbuch Pädagogische Psychologie (S. 804-812). Weinheim: Beltz/PVU.

Stamm, M. (2005a). Zwischen Exzellenz und Versagen. Frühleserinnen und Frührechner werden erwachsen. Chur/Zürich: Rüegger.

Stamm, M. (2005b). Bildungsaspiration, Begabung und Schullaufbahn: Eltern als Erfolgspromotoren? Schweizerische Zeitschrift für Bildungswissenschaften, 27, 277-295.

Stamm, M. (2005c). Hochbegabung und Schulabsentismus. Theoretische Überlegungen und empirische Befunde zu einer ungewohnten Liaison. Psychologie in Erziehung und Unterricht, 1, 20-33.

Stein, M. (2004). Berufliche Begabung erkennen. Paderborn: Eusl.

Supplee, P. L. (1990). Reaching the gifted underachiever. New York: Teachers College Press.

Taylor, R. D. (1994). Risk and resilience: Contextual influences on the development of African American adolescents. In M. C. Wang \& E. W. Gordon (Eds.), Educational resilience in inner city America (pp. 119-137). Hillsdale, NJ: Lawrence Erlbaum.

Wahl, D. (1975). Erwartungswidrige Schulleistungen. Weinheim: Beltz.

Weiner, I. B. (1992). Psychological disturbance in adolescence. New York: John Wiley and Sons.

Weinert, F. E. \& Petermann, F. (1980). Erwartungswidrige Schülerleistung oder unterdurchschnittlich determinierte Schulleistungen. In H. Heckhausen (Hrsg.), Fähigkeit und Motivation in erwartungswidriger Schulleistung (S. 19-52). Göttingen: Hogrefe.

Weiss, R. \& Osterland, J. (1980). Grundintelligenztest CFT 1. Braunschweig: Westermann.

Weiss, R. (1987). Grundintelligenztest CFT 20, Skala 2 mit Wortschatztest (WS) und Zablenfolgentest $(Z F)$. Göttingen: Hogrefe.

Whitmore, J. R. (1980). Giftedness, conflict, and underachievement. Boston: Allen \& Bacon.

Wild, E. \& Remy, K. (2002). Quantität und Qualität der elterlichen Hausaufgabenbetreuung von Drittklässlern in Mathematik. In M. Prenzel \& J. Doll (Hrsg.), Bildungsqualität von 
Schule: Schulische und ausserschulische Bedingungen mathematischer, naturwissenschaftlicher und überfachlicher Kompetenzen (S. 276-290). Weinheim: Beltz.

Schlagworte: Hochbegabung, Underachievement, Längsschnittstudie, Schulschwänzen, Lehrerbeziehungen.

\section{Underachievement. Un regard dans la boîte noire d'un phénomène irritant}

\section{Résumé}

Cet article présente les résultats d'une étude interrogeant les mauvaises performances des élèves doués. Basés sur les données de deux études suisses longitudinales, deux sous-groupes d'élèves (sous-performants et performants) ont été filtrés et testés par rapport à leurs caractéristiques socio-structurelles, leur attitude en classe et leur plan de carrière. Les "sous-performants » ont été définis comme des jeunes au QI $\geq 90$ avec des performances scolaires (rang, en pourcentage) en allemand et mathématiques $\geq 50$, les "performants» ont un QI $\geq 90$ et des performances scolaires en allemand et mathématiques $\geq 75$ ). Les résultats principaux montrent clairement des différences entre les performants et les sous-performants. Les sous-performants sont en premier lieu des jeunes gens issus de familles de la classe moyenne, moins motivés à réussir et qui ont de grands problèmes à s'autoréguler. Ils manquent d'autre part plus fréquemment l'école et ont clairement de plus mauvaises relations avec leurs professeurs que les élèves performants. Ces résultats montrent donc la nécessité de regarder les élèves sousperformants non pas uniquement dans une perspective individuelle, mais aussi dans une perspective institutionnelle.

Mots clés: haut potentiel, sous-performance (Underachievement), études longitudinales, absentéisme scolaire, relations avec le professeur

\section{Underachievement. Uno sguardo nella black-box di un fenomeno irritante}

\section{Riassunto}

Questo articolo presenta i risultati di uno studio che si interroga circa i risultati insoddisfacenti di allievi particolarmente dotati. Due studi longitudinali hanno fornito i dati relativi a due gruppi di allievi dotati sopra la media con riferimento alle loro caratteristiche socio-strutturali, l'attitutdine in classe e il piano di carriera. Dei due gruppi, uno era definito in base ad un $\mathrm{QI} \geq 90$ e prestazioni scolastiche in matematica e tedesco corrispondenti ad un rango $\geq 50$ e l'altro con un $\mathrm{QI} \geq 90$ e prestazioni scolastiche in matematica e tedesco corrispondenti ad un 
rango $\geq 75$. I risultati mostrano delle chiare differenze tra i due gruppi. Il primo gruppo dei «sottoperformanti» è formati di giovani maschi provenienti da famiglie di classe media, meno motivati e con problemi di auroregolazione. Inoltre marinano più sovente la scuola e evidenziano maggiori difficoltà nei rapporti con i docenti. Si tratta di risultati che richiamano alla necessità di considerare gli allievi con prestazioni insoddisfacenti rispetto al loro potenziale non solo da un punto di vista individuale ma anche istituzionale.

Parole chiave: risorse elevate, underachievement, studi longitudinali, assenteismo, relazioni con gli insegnanti

\title{
Underachievement. A glance in the black-box of an irritating phenomenon
}

\begin{abstract}
This article presents the results of a study on gifted underachievers. Based on the data of two sub-projects of Swiss longitudinal studies, two subgroups (underachievers and achievers) were extracted and tested for social-structural attributes, school attitudes and career plans. The group of the underachievers is defined as juveniles with an IQ percentage $\geq 90$ and a school achievement percentage in German/Mathematics $\geq 50$, the achievers with an IQ percentage $\geq 90$ and a school achievement percentage in German/Mathematics $\geq 75$. The main results point clearly to differences between achievers and underachievers. Underachievers are mainly male, mostly from middle-class families, less performance motivated and with considerable problems in self-regulation. They skip school more often, and they report on worse relationships to their teachers, than the achievers do. The results herewith point to the necessity of looking at underachievement not only from an individual, but also from an institutional perspective.
\end{abstract}

Key words: giftedness, underachievement, longitudinal study, truancy, teacher relationship 